\title{
Utilización de técnicas Kanban para la gestión de tesis doctorales
}

\section{T. Boronat, N. Montanes, D. Garcia-Sanoguera, O. Fenollar, V. Fombuena}

Grupo de Innovación de Prácticas Académicas (GIPA), Universitat Politècnica de València, Plaza Ferrándiz y Carbonell s/n, Alcoy, Alicante (ESPAÑA).

\begin{abstract}
The development of a doctoral thesis is a team work to be done by the doctorate and his supervisors. A huge list of jobs have to be faced by both. So, communication and coordination between them is mandatory in order an adequate support and track of the doctorate work is provided.

This job presents how Kanban technique is applied in the development of a doctoral thesis. The Kanban techniques allow to organize tasks through boards, in a very visual and simple way which facilitates the communication between doctorate and the directors. Recently web and mobile applications that implement Kanban techniques have appeared. These applications allow a dalily collaboration in the thesis, they allow to assign tasks, to know the state of its progress, to hold conversations, to provide constant feedback and finally to see which tasks have already been completed. In a short time, a large part of emails, telephone conversations and chats to notify the status of a task have been eliminated. In a short time, a large part of emails, phone conversations and chats to notify the status of a task have been eliminated.
\end{abstract}

Keywords: Teamwork, Trello, multiplatform tool, Learning monitoring

\section{Resumen}

A pesar de lo que pueda parecer, el desarrollo de una tesis doctoral no es un trabajo individual que debe realizar el doctorando, es un trabajo en equipo con los supervisores. Obliga al estudiante de doctorado y a los directores a hacer frente a una enorme lista de tareas para completar y recordar. Es necesario una coordinación y comunicación entre ellos para que los directores proporcionen un apoyo adecuado y rastreen el progreso del trabajo del estudiante.

Este trabajo propone la utilización de las técnicas Kanban para la gestión de una tesis doctoral. Las técnicas Kanban permiten organizar tareas a través de tableros, de una manera muy visual y simple lo que facilita la comunicación entre doctorando y directores. Últimamente han aparecido aplicaciones web y móvil que implementan las técnicas Kanban y por lo tanto permiten colaborar diariamente de manera virtual en la tesis, permiten asignar tareas, conocer el estado de su progreso, mantener conversaciones, proporcionar retroalimentación constante y finalmente ver aquellas tareas que ya se han completado. En poco tiempo se puede prescindir de una gran parte de correos electrónicos, conversaciones telefónicas y chats para notificar el estado de una tarea. 
Palabras clave: Kanban, tesis doctoral, trabajo en equipo, monitoreo del aprendizaje

\section{Introducción}

El desarrollo de una tesis doctoral es una experiencia para lograr la más alta calificación que la educación superior puede aportar. Una tesis es también una oportunidad para aplicar los conocimientos y habilidades adquiridos durante años en los estudios de grado y máster. El desarrollo de una tesis no es una cuestión banal, puede ser frustrante ya que se requiere para hacer una cantidad considerable de trabajo y el estudiante tiene que asumir su propia responsabilidad para la gestión de su aprendizaje. El trabajo a realizar durante el doctorado no está establecido por otros. Se espera que el doctorando inicie discusiones, pida ayuda cuando sea necesario, discuta acerca de lo que debería estar aprendiendo y así sucesivamente. El doctorando debe autogestionarse y debe decidir que debe ser capaz de planificare su trabajo, esto se debe identificar como una oportunidad no como una deficiencia.

Una tesis de doctoral es un proyecto de investigación individual, pero no debe ser completado completamente solo por el doctorado, el estudiante tiene la orientación de al menos un supervisor académico. El director de tesis debe tomar la iniciativa para obtener los recursos físicos para el correcto desarrollo del trabajo, el tema de investigación debe estar claramente definido para encajar en el impulso innovador del programa de investigación del supervisor que establecerá los límites al nivel de creatividad de investigación. Los desarrollos obtenidos son una copropiedad del supervisor y del estudiante de doctorado. Existe un gran interés tanto por el estudiante como por el supervisor de que el trabajo siga adelante para publicar trabajos conjuntos. A pesar de que la propiedad de los resultados es conjunta debe haber suficiente autonomía para que el estudiante pueda hacer una contribución original que justifique la concesión del doctorado.

Últimamente la supervisión de un estudiante no depende de un único supervisor. Las tesis doctorales son de tal envergadura que suelen afectar a más de un área de conocimiento por lo que es necesario disponer de un especialista de los diferentes temas afectados en el estudio. Los equipos de supervisores se establecen con un supervisor principal y uno o dos supervisores asociados.

Para gestionar el trabajo colaborativo entre el doctorado y los supervisores es necesario utilizar alguna herramienta o protocolo que facilite la interacción entre los miembros. En este trabajo se propone utilizar una herramienta de gestión de proyectos basada las técnicas Kanban. La herramienta permite colaborar diariamente de manera virtual en la tesis, permite asignar tareas, conocer el estado de su progreso, mantener conversaciones, proporcionar retroalimentación constante y finalmente ver aquellas que ya se han completado.

(cc) EY-NC-ND 2017, Universitat Politècnica de València 


\section{Objetivos}

El objetivo del presente estudio es presentar la metodología Kanban para la gestión de una tesis doctoral. Las técnicas Kanban se basa en un sistema de tarjetas desarrollado por Toyota con el objetivo de mejorar la eficiencia del proceso de fabricación de vehículos. Jim benson y Tonianne DeMaria Barry lo adecuaron para ser utilizado por equipos de trabajo e individuos. La adaptación la denominaron "Kanban personal".

Por otro lado, la herramienta presentada también fomenta el trabajo en equipo entre los miembros de una tesis doctoral (directores y doctorando) de forma que se favorezca la cooperación y un ambiente de confianza mutua entre los componentes.

\section{Desarrollo}

En los últimos tiempos, el uso de dispositivos tecnológicos ha invadido todas las facetas de la vida, incluida la educación y la gestión de proyectos. Existe un gran mercado de aplicaciones para cualquier propósito. Existen varias aplicaciones que permiten la aplicación de las técnicas Kanban de gestión de proyectos entre las que destacan: Kanbachi, Kanbanflow, Archmule, Boards, Asana y Trello. El presente trabajo muestra la utilización de Trello como herramienta que aplica las técnicas Kanban personales y su aplicación a la gestión de un proyecto singular como es una tesis doctoral.

Trello se basa en el paradigma de administración de proyectos Kanban. Kanban (kan japonés, que significa visual y prohibición, que significa tarjeta o tablero) es un sistema de información que controla armónicamente un proceso de producción, como la producción de un producto o la realización de una tesis doctoral. En este sistema visual, las tarjetas, que contienen información sobre cada uno de los procesos necesarios para alcanzar el objetivo, actúan como testigos del flujo de trabajo. Trello ofrece funcionalidades para la comunicación entre todos los componentes de un equipo, la definición de tareas y su asignación a los miembros. Además, permite compartir la información necesaria para llevar a cabo la tarea asignada y los resultados obtenidos asociados con la tarea ejecutada.

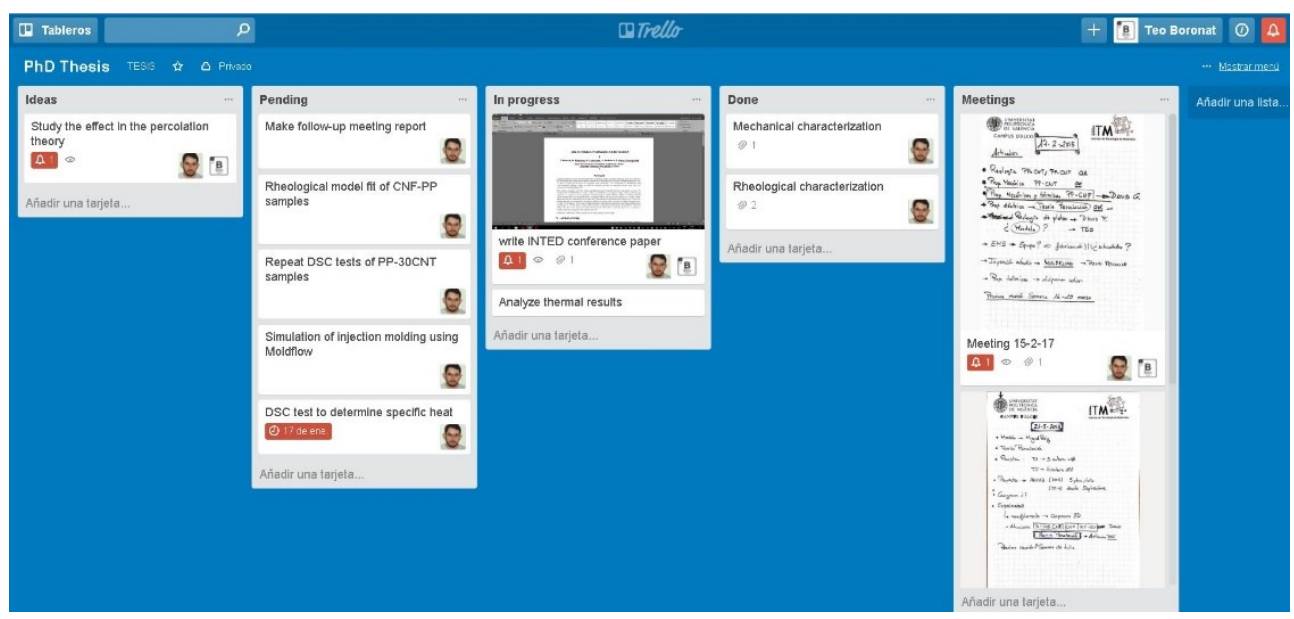

Fig. 1 Escritorio de Trello 
En Trello los proyectos están representados por paneles, que contienen listas de tareas. Una tesis doctoral será un proyecto. Estas listas contienen tarjetas (correspondientes a tareas en las que se puede subdividir una tesis). La figura 1 muestra el panel para una tesis con varias listas de tareas. Las tarjetas se pueden mover de una lista a otra, reflejando, por ejemplo, el flujo de una tarea desde la concepción hasta la planificación hasta que se ha ejecutado. Cada tarjeta (ver Figura 2) permite asignar diferentes atributos de una tarea, tales como:

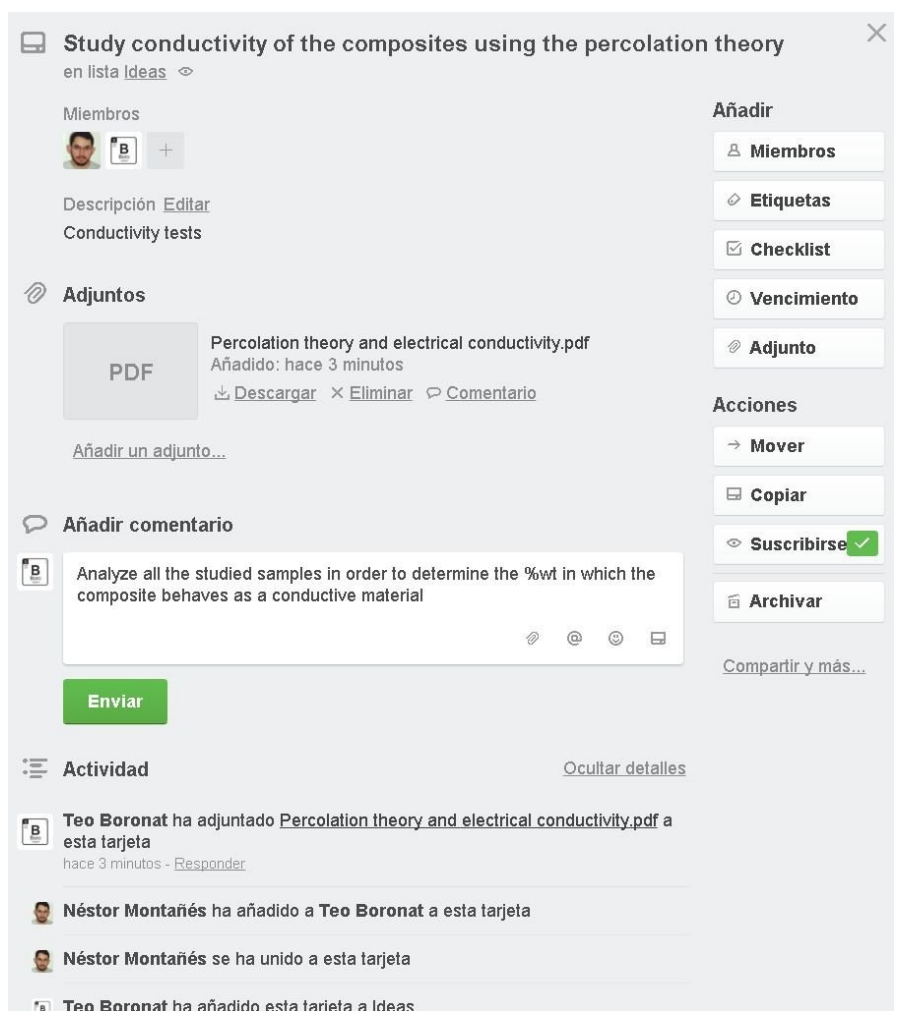

Fig. 2 Tarjeta de una tarea de Trello

La herramienta Trello se ha utilizado como plataforma colaborativa para la gestión de una tesis doctoral en de la Escuela de Doctorado de la Universitat Politècnica de València. El desarrollo de una tesis doctoral en el campo de la ingeniería es eminentemente práctico. Al inicio de la tesis doctoral se le mostró al alumno la herramienta Trello y se explican las principales funcionalidades a utilizar en el trabajo diario.

La gestión de la tesis se divide en varias etapas como "estado del arte", "experimental", "redacción". La etapa experimental, que es la principal, se divide en varias etapas más pequeñas ya que es muy extensa. Para cada etapa, los supervisores definen las actividades a realizar, los resultados o entregables a obtener y una estimación de la dedicación que debe realizar el doctorando.

Desde la primera etapa el doctorado configura su panel de trabajo como mejor le convenga. Como Trello es una herramienta altamente configurable, el doctorado puede tomar el manejo de diferentes maneras. A partir de la experiencia se ha podido comprobar que los 
estudiantes de doctorado generalmente organizan sus "Tarjetas" (las tareas a realizar) siguiendo dos estrategias principales:

- Crear una lista de tareas para cada etapa. Los estudiantes que siguen esta estrategia crean una lista con todas las tareas que deben realizarse para cada una de las etapas. El estado de cada tarea (creada, asignada, en proceso, en espera de aprobación, en revisión o finalizada) se indica mediante el uso de las "Etiquetas" proporcionadas por la herramienta. Al final de la tesis, todas las tareas se etiquetan como completadas.

- Distribuya el panel a un número fijo de listas de tareas. Los estudiantes que siguen esta estrategia usualmente crean al menos tres listas, llamadas pendientes, en progreso, y hechas. Sin embargo, algunos estudiantes crean otras listas, como revisar o aprobar por el supervisor. En esta estrategia, las tareas se trasladan de una lista a otra, siguiendo una ruta que comienza en la lista de tareas de la izquierda y termina en la lista a la derecha del panel. Las etiquetas se utilizan para clasificar las tareas en grupos: editar, repetir, memoria, presentación final, etc. Al final del curso, los alumnos han podido mover todas las tareas a la lista de tareas realizadas.

Independientemente de la estrategia elegida, durante el desarrollo de la tesis, los supervisores pueden ver cómo está progresando el trabajo. Usando la función "Adjuntar un archivo", el doctorando puede vincular a una tarjeta los resultados parciales de la tarea o la entrega final de una etapa. Trello ofrece la posibilidad de vincular un archivo desde una carpeta que todos los miembros del equipo pueden compartir en servicios externos como Google Drive o Dropbox.

La suscripción del supervisor a los paneles de trabajo del doctorado hace posible detectar la necesidad de algún tipo de apoyo o guía para seguir avanzando. El procedimiento que se define al principio de la tesis para que los alumnos puedan indicar la necesidad de ayuda es el siguiente:

- Los estudiantes asignan al supervisor a la tarjeta de la tarea sobre la cual tienen el problema. La herramienta envía un mensaje automatizado al profesor, informándole que el doctorando tiene una pregunta que hacer acerca de una tarea específica.

- El supervisor recibe el mensaje y accede a la tarjeta de tareas. Una vez que se ha consultado la descripción del problema y en caso de que sea necesario adjuntar con la resolución de la tarea, el supervisor realiza sus contribuciones mediante la función "Escribir un comentario" en la tarjeta de tareas. El doctorado recibe un mensaje automatizado indicando que el miembro supervisor ha hecho un comentario sobre la tarea.

- Por último, el alumno accede a la tarjeta, modifica su estado (cambiando su etiqueta) y elimina la asignación al supervisor de la tarea en caso de que no sea necesaria otra intervención por su parte. 


\section{Resultados}

La experiencia de utilizar las técnicas Kanban y su aplicación con la herramienta Trello en el campo de la enseñanza ha sido muy satisfactoria tanto por el supervisor como por el doctorado participante.

A continuación, se presentan algunas de las reflexiones ofrecidas por los participantes en esta iniciativa.

\subsection{Supervisor}

El principal beneficio observado por los autores de este artículo es que el supervisor puede tener un contacto directo y continuo con el doctorado incluso cuando está en una estancia en el extranjero. Este contacto permite:

- Conocer en cualquier momento el progreso de la obra encomendada. El profesor puede acceder a los paneles de trabajo del estudiante para saber si la dedicación planeada para realizar las tareas asignadas es suficiente. También permite saber qué tareas duran más y aquellas que requieren menos esfuerzo.

- Asegurar que la tesis esté al día. El supervisor puede corroborar que el estudiante no está perdido o está divagando sobre algún aspecto trivial.

- Trello le permite resolver un problema específico. El supervisor puede establecer directrices para los estudiantes de doctorado puede avanzar su trabajo.

\subsection{Doctorando}

A partir de la retroalimentación de los estudiantes que han utilizado la herramienta Trello se puede afirmar que están satisfechos con su uso como una plataforma de apoyo para el trabajo en equipo. Estas son algunas de las razones:

- Permite tener una visión clara y estructurada de todo el trabajo relacionado con la tesis. No sólo el trabajo ya realizado, también el trabajo pendiente.

- Permite centralizar todos los resultados parciales obtenidos en una sola plataforma.

- Evita el mal uso o la pérdida de documentos.

\section{Conclusiones}

Trello es una herramienta bien pensada, diseñada y probada que cubre los requisitos que una plataforma integrada para la coordinación y comunicación de los equipos de trabajo debe cumplir.

En este proyecto piloto no se pretendía solamente, pero no lo suficiente, proporcionar a los estudiantes una herramienta útil para llevar a cabo su trabajo de manera eficiente. También se buscó una forma de racionalizar el proceso de seguimiento de la investigación estudiantil, mecanizando algunas tareas básicas de validación de entrega de resultados parciales.

(cc) EY-NC-ND 2017, Universitat Politècnica de València 
La simplificación del proceso de seguimiento de los estudiantes da como resultado un ahorro de tiempo que puede ser utilizado para proporcionar una retroalimentación específica y adaptada a las necesidades específicas del doctorado.

Uno de los problemas planteados por los autores fue si la herramienta sería aceptada por los estudiantes. En ningún caso se pretendía que el aprendizaje de Trello supusiera un gran esfuerzo, ya que ese no es el objetivo principal de la tesis. Debe tenerse en cuenta que no ha sido detectadoningún problema derivado del uso de la herramienta, en gran parte, por su interfaz simple, clara e intuitiva

\section{Referencias}

BENSON, J y DEMARIA BARRY, T. (2011) Personal Kanban. LondresCreatespace

DELGADO, A.,LLUIS-MESQUIDA, A. Y MAS, A. (2014) "Utilización de Trello para realizar el seguimiento del aprendizaje de equipos de trabajo". López, D. (coord.) En: Actas del Simposio/Taller XX JENUI. Oviedo: JENUI. 769-822

JAPAN MANAGEMENT ASSOCIATION (1998) Kanban y Just In Time en Toyota.la direccion empieza en las estaciones. Prod. Press

TRAVER, V.J., TRAVER, J.A. (2004) “iPor qué no enseñamos a aprender cooperativamente?”. ”. López, D. (coord.) En: X Jornadas de Enseñanza Universitaria de la Informática (X JENUI). Alicante: JENUI. 297-304

Página web de Trello. $<$ https://trello.com/> [Consulta: 2 de abril de 2017] 